\title{
Theoretical Developments on Hard QCD Processes at Colliders *
}

\author{
Thomas Gehrmann \\ Institut für Theoretische Physik, RWTH Aachen, D-52056 Aachen
}

\section{Introduction}

Quantum Chromodynamics (QCD) is well established as theory of strong interactions through a large number of experimental verifications. The era of 'testing QCD' is clearly finished, and QCD today is becoming precision physics. In contrast to LEP and SLC, where electroweak reactions could be studied to a highly precise level without having to take QCD effects into account, QCD is ubiquitous at hadron colliders, affecting all observables. Any precision measurement (strong coupling constant, quark masses, electroweak parameters, parton distributions) at the Tevatron and the LHC, as well as any prediction of new physics effects and their backgrounds, relies on the understanding of QCD effects on the observable under consideration.

Precision QCD poses several severe challenges from the theoretical and computational point of view. Most importantly, the strong coupling constant is considerably larger than the electromagnetic coupling constant at scales typically probed at colliders: $\alpha_{s}\left(M_{Z}\right) \simeq 15 \alpha_{\mathrm{em}}\left(M_{Z}\right)$, resulting in a slower convergence of the perturbative expansion. As a consequence, a precise description of QCD observables (precise means here that the theoretical uncertainty becomes similar to the achieved or projected experimental errors) is obtained only by including higher order corrections, often requiring beyond the next-to-leading order. Another important challenge is the fact that QCD describes quarks and gluons, while experiments observe hadrons. This mismatch is either accounted for by a description of the parton to hadron transition through fragmentation functions or by defining sufficiently inclusive final state observables, such as jets. Finally, many collider observables involve largely different scales, such as quark masses, transverse momenta and vector boson masses. These give rise to potentially large logarithms, which might spoil the convergence of the perturbative series and need to be resummed to all orders.

In this talk, I shall try to highlight recent theoretical progress towards precision QCD at colliders, focusing on heavy quark production in Section 2, on jets and multiparton final states in Section 3 and on photons and electroweak bosons in Section 1 . Finally, a summary of the current state-of-the-art and of yet open issues is given in Section 5 .

\footnotetext{
* Invited talk at the 14th Topical Conference on Hadron Collider Physics (HCP2002), Karlsruhe, Germany, 30 Sep-4 Oct 2002
} 


\section{Heavy Quarks}

The production of heavy quarks is one of the main topics investigated at high energy collider experiments. Heavy quarks are of particular interest to elucidate the flavour sector of the standard model, which is less well tested than the gauge sector. Also, many approaches to physics beyond the standard model, often related to electroweak symmetry breaking and mass generation, predict new effects to be most pronounced in observables involving heavy quarks.

\subsection{Total Cross Sections}

The total cross sections for the production of heavy quarks can be computed within perturbation theory. The current state-of-the-art is a next-to-leading order (NLO) calculation [1], which is further improved by summing large logarithms due to soft gluon emission up to the next-to-leading logarithmic (NLL) level [2]. As can be seen from Figure 1, these predictions are in good agreement with experimental data on the total $t \bar{t}$ cross section at the Tevatron [3] and the total $b \bar{b}$ cross section at HERA-B [4] (which both actually refer to similar kinematical values of $m_{Q} / \sqrt{s}$ ). The theoretical uncertainty on the prediction for HERA-B is larger for two reasons: the larger value of the strong coupling at $m_{b}$ than at $m_{t}$ and the dominance of $g g$ initial states in $p N$ collisions (HERA-B) compared to $q \bar{q}$ dominance in $p \bar{p}$ collisions (Tevatron).

\subsection{Transverse Momentum Distributions}

Differential distributions of hadrons containing $b$ quarks measured in hadronhadron, photon-hadron or photon-photon collisions have been in apparent discrepancy with theoretical predictions for quite some time. The spectrum of $B^{ \pm}$hadrons measured at $\mathrm{CDF}[\mathrm{F}$ is one of the most recent examples for this discrepancy.
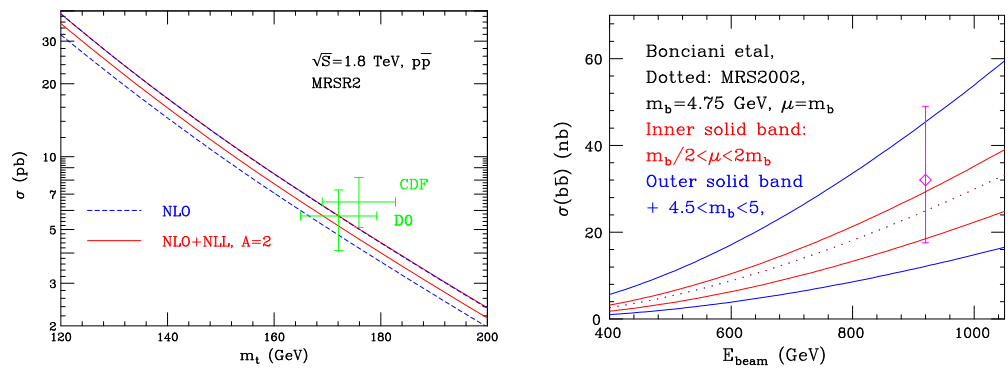

Fig. 1. Total cross sections for $t \bar{t}$ at the Tevatron and $b \bar{b}$ at HERA-B. 
The theoretical prediction for $B$ meson production involves a convolution of the hard matrix element for heavy quark production in parton-parton scattering with initial parton distributions and final state fragmentation functions describing the non-perturbative transition from a $b$ quark to a $B$ hadron. It is in particular the latter which may account for the discrepancy between theoretical prediction and experimental data, especially since it has been observed [6] that the transverse momentum distribution of $b$-tagged jets [7] (which has little sensitivity to fragmentation functions) is in much better agreement with theoretical predictions.

The definition of heavy quark fragmentation functions is not free from ambiguities, since some aspects of these functions are actually calculable in perturbation theory [8]. In extracting these fragmentation functions from data on $B$ hadron production in $e^{+} e^{-}$collisions, several choices are made, related to the order of perturbation theory, the incorporation of mass effects in the matrix elements, the resummation of potentially large perturbative terms, the correction of data for parton showers or the parametric form of the ansatz used in the determination. A commonly used parametric form is the so-called Peterson ansatz [9], which contains only a single parameter $\epsilon$, describing the shape of the non-perturbative fragmentation function. Unfortunately, the sensitivity of the fragmentation function on the assumptions used in the extraction from $e^{+} e^{-}$spectra is often overlooked when using this fragmentation function to compute heavy hadron spectra at colliders. It was pointed out in 10] that a consistent treatment (in this case a purely massless approach) in extracting and implementing fragmentation functions can yield a sizable reduction of the discrepancy between experimental data and theoretical prediction.

In the kinematical range covered by the heavy hadron production at Tevatron, $b$ quark mass effects are sizable [11. An approach incorporating quark mass effects, perturbatively calculable components of the heavy quark fragmentation function [8] and resummation of large logarithms up to the next-to-leading logarithmic level was presented in [11] with the fixed-order next-to-leading log (FONLL) scheme, which requires only a small, genuinely non-perturbative component of the fragmentation function to be fitted to $e^{+} e^{-}$data. In order to expose the information content actually relevant to heavy hadron spectra at hadron colliders, this fit is done in moment space.

In view of new data from ALEPH [12], a phenomenological study of $B$ hadron production at colliders based on the FONLL scheme [11] was presented in [13]. It was shown that the consistent treatment of the fragmentation function in extraction and prediction reduced the discrepancy between data and theoretical prediction considerably, Figure 2. The theoretical prediction is however still falling somewhat short of the experimental data, which is probably due to currently uncalculated corrections beyond NLO.

Many collider experiments also report an excess in the $b$ quark production spectra. In interpreting these data, it must always be kept in mind that 


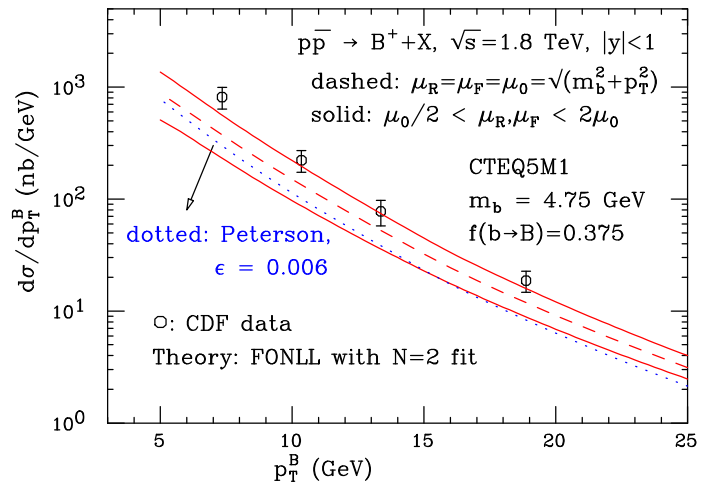

Fig. 2. Transverse momentum spectrum of $B$ hadrons at CDF, compared to calculations using Peterson and FONLL fragmentation functions, from [13].

it is not $b$ quark but $B$ hadron production which is observed in the experiment. Information on $b$ quark production is only inferred from these data using some model for the heavy quark fragmentation. As discussed above, there are numerous ambiguities, which can yield inconsistent predictions if not implemented consistently. In view of the rather sizable effects due to a consistent treatment of the fragmentation function observed on the $B$ hadron spectra at CDF, it might be that the data sets on $b$ quark spectra have to be reanalysed incorporating the new experimental information on the $b$ quark fragmentation functions in a consistent manner.

\subsection{Top Quark Spin Correlations}

Contrary to $b$ and $c$ quarks, which have lifetimes much longer than the typical timescale required for hadronization, $t$ quarks are too short-lived to hadronize before they decay. As a consequence, top quark production and decay can be fully computed within perturbative QCD, and the spin state of the $t$ quark determined from the distribution of its decay products [14] reflects the $t$ quark spin induced in the production process, giving rise to non-trivial spin correlations in $t \bar{t}$ pair production processes 15]. At present, data from Tevatron Run I are not yet sufficiently precise to probe these spin correlations, results obtained up to now concern only the polarization state of the vector boson produced in the $t$ decay [16]. Future measurements at Run II and LHC will allow to use $t \bar{t}$ spin correlations as a sensitive probe of potential effects beyond the standard model showing up in the electroweak vertex of $t$ decay.

\section{Jets}

Hadronic jets at large transverse momenta are produced very copiously at colliders. Final states with a small number of jets are measured to very high 


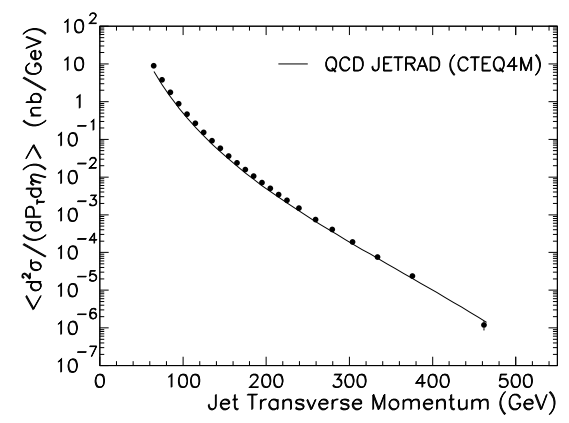

Fig. 3. Single jet inclusive cross section at NLO compared to D0 data 22].

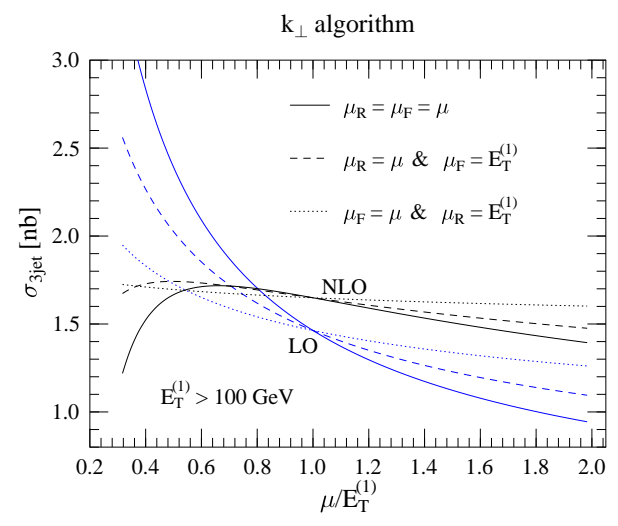

Fig. 4. Dependence of the NLO prediction for the $3 j$ cross section at the Tevatron on renormalization and factorization scale, from [19].

experimental accuracy, such that they can be used for precision measurements of the strong coupling constant and of parton distribution functions. Multiparton final states, involving a large number of jets, can on the other hand mimic final state signatures introduced by physics beyond the standard model, thus forming an irreducible background to searches.

The current state of the art for the theoretical description of jet observables at hadron colliders is next-to-leading order QCD. To this order, parton level event generators are available for $1 j+X$ (EKS [17] and JETRAD [18]), $2 j+X$ (JETRAD [18]), $3 j+X$ (NLOJET $++\sqrt{19}])$ and for $V+X, V+1 j+X$ (both DYRAD 201),$V+2 j+X$ (MCFM 21]). In particular, the MCFM project aims to provide a library of a large number of final states to NLO accuracy. Final states with more than three identified particles (vector bosons or jets) are at present only known to leading order. NLO predictions do in general yield a very good description of the experimental data, e.g. for the $1 j$ inclusive cross section [22], Figure 3. The uncertainty on the theoretical 
prediction, as estimated by varying renormalization and factorization scales, is reduced considerably from LO to NLO, Figure 1 . Nevertheless, for observables such as the single jet inclusive cross section, the theoretical errors of the NLO prediction are still in excess of the experimental errors.

\subsection{Jet Definitions}

Jets in high energy physics experiments are identified by applying a jet algorithm to hadronic final states. The default jet algorithm used in the analysis of Tevatron Run I data (iterative fixed cone algorithm) turned out to be unsuited for the study of multi-jet cross sections and to be infrared unsafe if a theoretical implementation beyond NLO is attempted. These problems can essentially be truncated back to difficulties in splitting and merging neighbouring cones, which is done using an ad-hoc separation parameter. Several improved algorithms have been suggested, which can be grouped into two classes: the $k_{T}$ type algorithm [23] uses a clustering procedure combining neighbouring preclusters according the transverse momentum of each cluster and to their mutual spatial separation. The improved legacy cone algorithm [24] is an improved version of the iterative fixed cone algorithm, which avoids the problems of the latter.

\subsection{Precision Jet Physics}

Despite the evidently good agreement of NLO QCD with experimental data on jet production rates, Figure 3, predictions to this order are insufficient for many applications. For example, if one uses data on the single jet inclusive cross section 25] to determine the strong coupling constant $\alpha_{s}$, it turns out that the dominant source of error on this extraction is due to unknown higher order corrections. Given that the theoretical prediction to infinite order in perturbation theory should be independent of the choice of renormalization and factorization scale, this error can be estimated from the variation of the extracted $\alpha_{s}$ under variation of these scales, as seen in Figure 5 . As a result, CDF find from their Run I data

$$
\alpha_{s}\left(M_{Z}\right)=0.1178 \pm 0.0001(\text { stat })_{-0.0095}^{+0.0081}(\text { sys })_{-0.0047}^{+0.0071}(\text { scale }) \pm 0.0059 \text { (pdf). }
$$

It can be seen that the statistical error is already negligible; improvements in the systematic error can be anticipated in the near future. To lower the theoretical error, it is mandatory to compute next-to-next-to-leading order (NNLO) corrections to the single jet inclusive cross section.

A similar picture is true in $e^{+} e^{-}$annihilation into three jets and deep inelastic $(2+1)$ jet production, where the error on the extraction of $\alpha_{s}$ from experimentally measured jet shape observables [26] is completely dominated by the theoretical uncertainty inherent in the NLO QCD calculations.

Besides lowering the theoretical error, there is a number of other reasons to go beyond NLO in the description of jet observables [27]. While jets at 


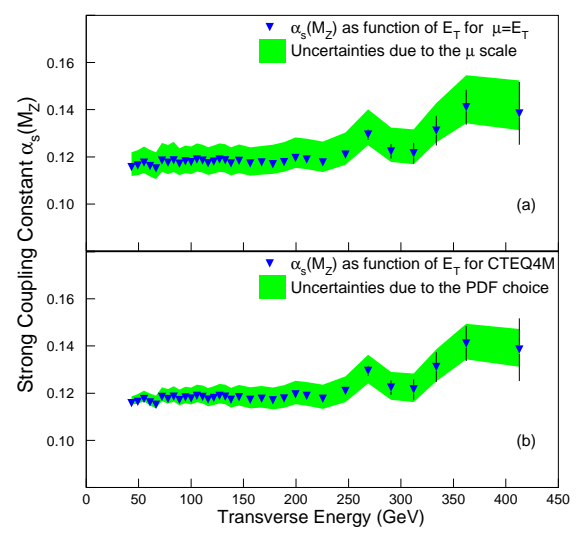

Fig. 5. Errors on extraction of $\alpha_{s}$ from single jet inclusive cross section at CDF 25].

NLO are modelled theoretically by at most two partons, NNLO allows up to three partons in a single jet, thus improving the matching of experimental and theoretical jet definitions and resolving the internal jet structure. At hadron colliders, NNLO does also account for double initial state radiation, thus providing a perturbative description for the transverse momentum of the hard final state. Finally, including jet data in a global NNLO fit of parton distribution functions, one anticipates a lower error on the prediction of benchmark processes at colliders.

The calculation of jet observables at NNLO requires a number of different ingredients. To compute the corrections to an $n$-jet observable, one needs the two-loop $n$ parton matrix elements, the one-loop $n+1$ parton matrix elements and the tree level $n+2$ parton matrix elements. Since the latter two contain infrared singularities due to one or two partons becoming theoretically unresolved (soft or collinear), one needs to find one- and two-particle subtraction terms, which account for these singularities in the matrix elements, and are sufficiently simple to be integrated analytically over the unresolved phase space. One-particle subtraction at tree level is well understood from NLO calculations [28] and general algorithms are available for one-particle subtraction at one loop [29]. Tree level two-particle subtraction terms have been studied in the literature [30], their integration over the unresolved phase space was up to now made only in one particular infrared subtraction scheme in the calculation of higher order corrections to the photon-plus-one-jet rate in $e^{+} e^{-}$annihilation [31]. A general two-particle subtraction procedure is still lacking at the moment, although progress on this is anticipated in the near future.

Concerning virtual two-loop corrections to jet-observables related to $2 \rightarrow$ 2 scattering and $1 \rightarrow 3$ decay processes, enormous progress has been made in the past two years. Much of this progress is due to several technical develop- 


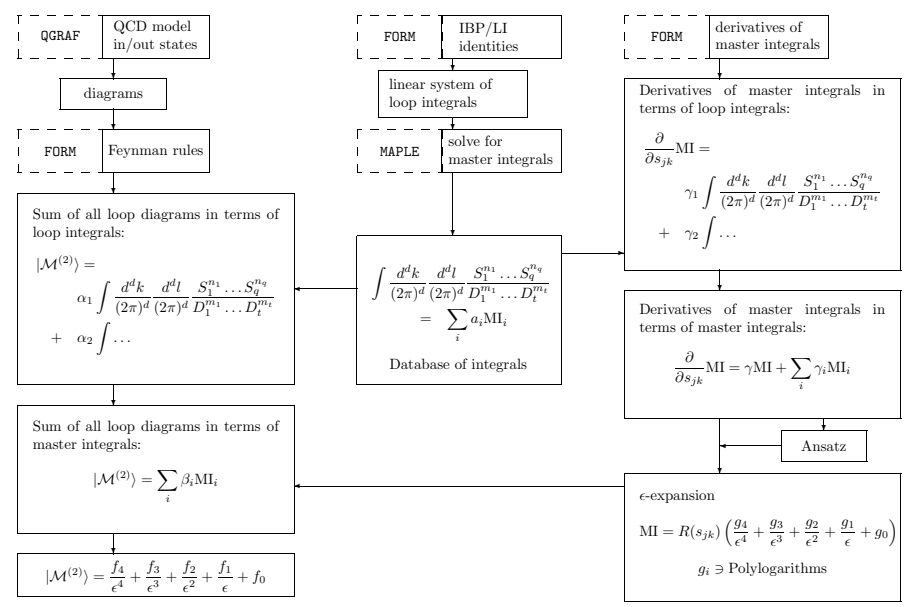

Fig. 6. Computer algebra to compute two-loop scattering matrix elements for jet physics.

ments concerning the evaluation of two-loop multi-leg integrals. Using iterative algorithms [32], one can reduce the large number of two-loop integrals by means of integration-by-parts [33] and Lorentz invariance [34 identities to a small number of master integrals. The master integrals relevant to two-loop jet physics are two-loop four-point functions with all legs on-shell [35] or one leg off-shell [36], which were computed using explicit integration or implicitly from their differential equations [34].

Combing the reduction scheme with the master integrals, it is straightforward to compute the two-loop matrix elements relevant to jet observables using computer algebra 37]. The generic structure of such a calculational procedure is depicted in Figure 6. Following this procedure, two-loop matrix elements were obtained for Bhabha scattering [38], parton-parton scattering into two partons [39], parton-parton scattering into two photons [40], as well as light-by-light scattering 41. Most recently, two-loop corrections were computed for the off-shell process $\gamma^{*} \rightarrow q \bar{q} g$ 42, relevant to $e^{+} e^{-} \rightarrow 3 j$. Part of these results were already confirmed 433] using an independent method 444. Related to $e^{+} e^{-} \rightarrow 3 j$ by analytic continuation [45] are $(2+1) j$ production in ep collisions and $V+j$ production at hadron colliders. A strong check on all these two-loop results is provided by the agreement of the singularity structure with predictions obtained from an infrared factorization formula 46 .

\subsection{Multiparton Processes}

Many scenarios for physics beyond the standard model predict visible signals only in observables with a large number of partons in the final state due to production and subsequent decay of yet unknown short-lived particles. The 
same multi-parton final states can however also be produced through ordinary QCD processes, acting as a background to the new physics signals. The extraction of new physics effects from multiparton final states does therefore require predictions for QCD backgrounds for these final states.

It turns out that a diagrammatic evaluation of the corresponding matrix elements (even using modern techniques such as helicity amplitudes) becomes rapidly infeasible due to the factorial increase in the number of Feynman diagrams with increasing number of final state particles. An alternative approach 477 circumvents the need for a direct evaluation of Feynman diagrams by determining scattering matrix elements directly from the numerical Legendre transformation of the interaction Lagrangian. This procedure yields only a power-like growth of computation time with increasing number of final state particles, and does therefore outpace the Feynman diagrammatic evaluation for high multiplicity final states. Extending earlier work 48 on final states with up to nine gluons, a parton level event generator ALPHGEN [49] to compute

- $W / Z / \gamma^{*}+Q \bar{Q}+(n \leq 4) j$

- $H+Q \bar{Q}+(n \leq 4) j$

- $W / Z / \gamma^{*}+(n \leq 6) j$

- $n W+m Z+l H+N j(n+m+l+N \leq 8, N \leq 3)$

- $Q \bar{Q}+(n \leq 6) j$

- $Q \bar{Q}+Q^{\prime} \overline{\bar{Q}}^{\prime}+(n \leq 4) j$

was presented recently. The event generation provides full colour and flavour information, such that a hadronization model and a subsequent detector simulation can be interfaced.

Combining matrix element based event generation with parton showers, special care has to be taken to avoid overcounting of contributions. Extending a method derived for $e^{+} e^{-}$annihilation [50], a procedure for reweighting of events and determining appropriate starting conditions and cut-off of the parton shower has been proposed [51] to allow combination with the exact multi-parton matrix elements.

A closely related issue is the incorporation of next-to-leading order corrections in parton shower Monte Carlo programs. Recently, algorithms were devised for this task by several groups [52], first programs implementing these algorithms have already been released [53].

\section{Photons and Massive Gauge Bosons}

Photons and gauge bosons provide very prominent final state signatures at colliders. Their study allows the precise determination of electroweak parameters at hadron colliders, and their final state signatures are often background to searches, such as photon pair production to the Higgs search in the lower mass range. 


\subsection{Isolated Photons}

Photons produced in hadronic collisions arise essentially from two different sources: 'direct' or 'prompt' photon production via hard partonic processes such as $q g \rightarrow q \gamma$ and $q \bar{q} \rightarrow g \gamma$ or through the 'fragmentation' of a hadronic jet into a single photon carrying a large fraction of the jet energy. The former gives rise to perturbatively calculable short-distance contributions whereas the latter is primarily a long distance process which cannot be calculated perturbatively and is described in terms of the quark-to-photon fragmentation function. In principle, this fragmentation contribution could be suppressed to a certain extent by imposing isolation cuts on the photon. Commonly used isolation cuts are defined by admitting only a maximum amount of hadronic energy in a cone of a given radius around the photon. An alternative procedure is the democratic clustering approach suggested in [54], which applies standard jet clustering algorithms to events with final state photons, treating the photon like any other hadron in the clustering procedure. Isolated photons are then defined to be photons carrying more than some large, predefined amount of the jet energy.

Both types of isolation criteria infrared safe, although the matching of experimental and theoretical implementations of these criteria is in general far from trivial. It was pointed out recently 55 that cone-based isolation criteria fail for small cone sizes $R$ (once $\alpha_{s} \ln R^{-2} \sim 1$ ), since the isolated photon cross section exceeds the inclusive photon cross section. This problem can only be overcome by a resummation of the large logarithms induced by the cone size parameter.

The contribution from photon fragmentation to isolated photon cross sections at hadron colliders is sensitive (for both types of isolation criteria) on the photon fragmentation function at large momentum transfer, which has up to now been measured only by one of the LEP experiments [56. Based on the democratic clustering procedure, ALEPH extracted the quark-to-photon fragmentation function from the measured photon-plus-one-jet rate. It was observed that the resulting prediction 54 for the variation of the isolated photon rate with the resolution parameter was in good agreement with the measurement, especially once NLO corrections [31] were included. A related OPAL measurement [57] of the photon fragmentation function from inclusive photon production in $e^{+} e^{-}$is unfortunately not sufficiently sensitive on the behaviour at large momentum transfers [58]. Further information on the photon fragmentation function at large momentum transfer might be gained from yet unanalysed LEP data or from the study of photon-plus-jet final states in deep inelastic scattering at HERA [59], where first data are now becoming available 60.

\subsection{Photon Pairs}

The discovery of a light Higgs boson $\left(m_{H} \lesssim 140 \mathrm{GeV}\right)$ at the LHC is based largely on the observation of the rare decay to two photons [61. To perform 

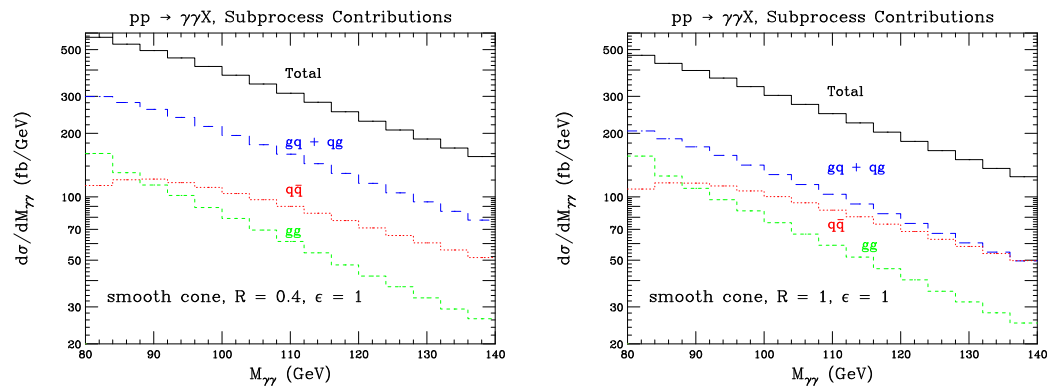

Fig. 7. Contributions from $q \bar{q}, q g$ and $g g$ subprocesses to $\gamma \gamma$ final states for different isolation criteria, from 63 .
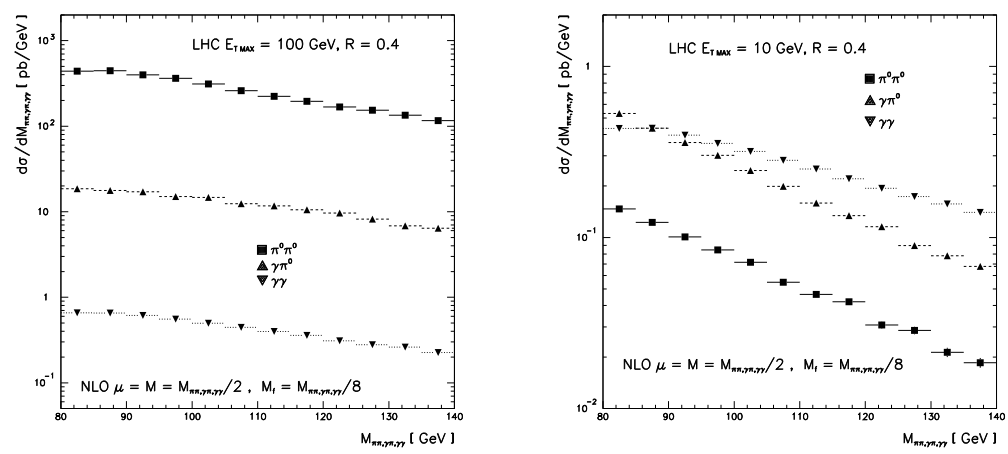

Fig. 8. Comparison of $\pi^{0} \pi^{0}, \pi^{0} \gamma$ and $\gamma \gamma$ production cross sections for different isolation criteria, from 65 .

an accurate background subtraction for this observable, one requires a precise prediction for QCD reactions yielding di-photon final states. At first sight, the $\mathcal{O}\left(\alpha_{s}^{0}\right)$ process $q \bar{q} \rightarrow \gamma \gamma$ yields the leading contribution. However, due to the large gluon luminosity at the LHC, both $q g \rightarrow q \gamma \gamma\left(\mathcal{O}\left(\alpha_{s}^{1}\right)\right)$ and $g g \rightarrow \gamma \gamma\left(\mathcal{O}\left(\alpha_{s}^{2}\right)\right)$ subprocesses yield contributions of comparable magnitude (Figure 7). The NLO corrections to the $q \bar{q}$ and $q g$ subprocesses have been known for quite some time, these are implemented in the flexible parton level event generator DIPHOX [62]. Most recently, NLO corrections were also derived for the $g g$ subprocess 63$]$. Since the lowest order contribution to this process is already mediated by a quark loop, this calculation contains some of the features appearing in jet physics only at NNLO, such as two-loop QCD amplitudes and unresolved limits of one-loop amplitudes (see Section 3.2 above).

It must be kept in mind that the di-photon cross sections are highly sensitive on the isolation criteria applied to the photons, Figure 7, with a sub- 
stantial contribution arising from photon fragmentation at large momentum transfers [64]. Moreover, it is experimentally difficult to distinguish photons from highly energetic neutral pions which decay into a closely collimated photon pair, mimicking a single photon signature. The pion background in photon pair production has been studied to NLO in [65] and implemented in DIPHOX, showing that in particular the $\pi^{0} \gamma$ channel remains comparable to the $\gamma \gamma$ channel even for tight isolation criteria, Figure 8 .

\subsection{Vector Boson and Higgs Production}

The production cross sections for $W^{ \pm}$and $Z^{0}$ bosons at hadron colliders are well understood both experimentally and theoretically. At present, these cross sections are measured to an error of about $10 \%$ from Tevatron Run I, largely limited by statistics. A considerable reduction of the experimental error is anticipated from Run II and for the LHC. On the theory side, inclusive vector boson production has been computed to NNLO 66 already more than ten years ago. Very recently, these results have been verified for the first time in an independent calculation 67. The uncertainty on the theoretical prediction has been assessed in detail in [68] and found to be around 3\% from variations of renormalization and factorization scale as well as from uncertainties on the parton distribution functions. Some improvements are anticipated once full NNLO determinations [69] of the parton distribution functions become available.

Given the good theoretical and experimental understanding of $W^{ \pm}$and $Z^{0}$ boson production, it has been suggested to use these for a determination of the LHC luminosity [70]. In practice, it turns out that it is not possible to measure the fully inclusive production cross sections, but only cross sections integrated over a restricted range in rapidity, which are only known to NLO at present [71. For the $W^{ \pm}$production, which is observed only through the $l \nu$ decay channel, it is moreover mandatory to compute the spatial distribution of the decay products, which is again only known to NLO [20]. To match the anticipated experimental accuracy at the LHC and to render vector boson production a reliable luminosity monitor, it would be required to extend both these calculations to NNLO.

Closely related to the inclusive vector boson production cross section is the inclusive Higgs boson production cross section in the infinite top mass limit. While the former is induced, at the leading order, by the partonic subprocess $q \bar{q} \rightarrow W^{ \pm}, Z^{0}$, the latter is $g g \rightarrow H$. The NNLO corrections to inclusive Higgs boson production have been derived, first in the soft/collinear approximation [72]; shortly thereafter, the full coefficient functions were obtained by expansion around the soft limit [67, and fully analytically [73] by extending the IBP/LI reduction method and the differential equation technique (see Section 3.2) to compute double real emission contributions. It turned out that inclusion of NNLO corrections yields a sizable enhancement of the Higgs production cross section, and a reduction of the uncertainty due 
to renormalization and factorization scale. These calculations were also extended to pseudoscalar Higgs production 74. A more detailed discussion of recent results on Higgs physics at colliders can be found in [61.

\subsection{Transverse Momentum Distributions}

Transverse momentum distributions of vector bosons or any other colourneutral final states are well described in perturbation theory [75] only if the transverse momentum $q_{T}$ is of the same order as the invariant mass $M$ of the colour-neutral final state. Otherwise, the convergence of the perturbative series is spoilt by large logarithms $\ln \left(q_{T} / M\right)$. Reliable predictions can be obtained if these logarithms are resummed to all orders in perturbation theory [76]. This resummation procedure exponentiates logarithms from three different sources: soft $(A)$ and collinear $(B)$ radiation associated to the hard interaction (expressed as Sudakov form factor) and collinear initial state radiation $(C)$. The resummation coefficients $A, B, C$ can be expanded in powers of the strong coupling constant; it turns out that the soft coefficient $A$ only depends on the partonic initial state, while $B, C$ also depend on the final state under consideration.

The leading logarithmic (LL) corrections are obtained from soft exponentiation alone, while collinear contributions first enter at the next-to-leading logarithmic (NLL) level [77. Finally, collinear initial state radiation affects only higher (NNLL) order logarithms. At present, NNLL resummed corrections are known for electroweak vector boson production [78] and Higgs production [79]. Incorporation of these corrections [80] improves the theoretical description of vector boson production at small transverse momenta considerably, and yields a stabilisation of the theoretical prediction under variations of renormalization and factorization scales.

The purely soft $(A)$ terms can be read off from the partonic splitting functions to the appropriate order 69] or computed by considering only eikonal diagrams 81.

By reordering the terms in the resummation formula, it is possible to extract the final-state independent terms in the $B$ and $C$ coefficients, and to group the genuinely process dependent terms into a hard coefficient function $H$. This modified resummation formula 82 allows to obtain resummed predictions for a large number of different final states (such as vector boson pairs) without having to rederive the full set of resummation coefficients.

In the course of the computation [83] of these universal resummation coefficients to NNLL accuracy, one encounters configurations similar to the ones appearing in double real radiation contributions to jet physics at NNLO.

Besides the above developments on extending the existing resummation formalism towards higher accuracy, considerable progress has been made also to perform resummation in processes not characterised by a colour neutral system with a large invariant mass. In particular, a formalism to jointly resum 
partonic threshold and recoil corrections has been developed 84 and applied successfully to prompt photon and vector boson production [85].

\section{Conclusions and Outlook}

QCD at present and future hadron colliders will be precision physics, very much like electroweak physics was precision physics in the LEP era. The study of many of the standard scattering reactions will allow a precise determination of the strong coupling constant, electroweak parameters, quark masses and parton distribution functions. In turn, this information translates in improved predictions for new physics signals and their backgrounds.

To match the experimental accuracy reached for a number of QCD collider observables, the current theoretical predictions have to be improved in several aspects. Most importantly, NLO QCD is often not enough if confronted with high precision data (such as for jets and gauge bosons) or observables with slowly converging perturbative expansions (such as heavy quarks or the Higgs boson). Considerable progress has been made very recently in extending QCD calculations to NNLO, first results on inclusive observables are now available, and calculations for jet observables are well advanced. Many observables do moreover require the resummation of large logarithms spoiling the convergence of the perturbative series. A universal picture for these resummations started to emerge recently, and new techniques have been developed to compute the corresponding resummation coefficients. Fragmentation effects enter many observables with identified particles in the final state. In particular, a consistent treatment of heavy quark fragmentation effects can account for a large part of the observed discrepancy in $B$ hadron spectra, and quark-to-photon as well as quark-to-pion fragmentation yield important contributions to photon pair final states forming an important background to Higgs searches. Much valuable information on these fragmentation functions is contained in data from LEP, and should be extracted (as long as this is still a feasible task) to improve predictions for collider observables.

\section{References}

1. P. Nason, S. Dawson and R. K. Ellis, Nucl. Phys. B 303 (1988) 607; W. Beenakker, W. L. van Neerven, R. Meng, G. A. Schuler and J. Smith, Nucl. Phys. B 351 (1991) 507.

2. R. Bonciani, S. Catani, M. L. Mangano and P. Nason, Nucl. Phys. B 529 (1998) 424 arXiv:hep-ph/9801375.

3. T. Affolder et al. [CDF Collaboration], Phys. Rev. D 64 (2001) 032002 arXiv:hep-ex/0101036; V. M. Abazov et al. [D0 Collaboration], arXiv:hepex/0205019.

4. I. Abt et al. [HERA-B Collaboration], arXiv:hep-ex/0205106.

5. D. Acosta et al. [CDF Collaboration], Phys. Rev. D 65 (2002) 052005 arXiv:hep-ph/0111359. 
6. B. Abbott et al. [D0 Collaboration], Phys. Rev. Lett. 85 (2000) 5068 arXiv:hep-ex/0008021.

7. S. Frixione and M.L. Mangano, Nucl. Phys. B 483 (1997) 321 arXiv:hep$\mathrm{ph} / 9605270$.

8. B. Mele and P. Nason, Nucl. Phys. B 361 (1991) 626.

9. C. Peterson, D. Schlatter, I. Schmitt and P.M. Zerwas, Phys. Rev. D 27 (1983) 105.

10. J. Binnewies, B. A. Kniehl and G. Kramer, Phys. Rev. D 58 (1998) 034016 arXiv:hep-ph/9802231.

11. M. Cacciari, M. Greco and P. Nason, JHEP 9805 (1998) 007 arXiv:hep$\mathrm{ph} / 9803400$.

12. A. Heister et al. [ALEPH Collaboration], Phys. Lett. B 512 (2001) 30 arXiv:hep-ex/0106051.

13. M. Cacciari and P. Nason, Phys. Rev. Lett. 89 (2002) 122003 arXiv:hep$\mathrm{ph} / 0204025$.

14. A. Brandenburg, Z. G. Si and P. Uwer, Phys. Lett. B 539 (2002) 235 arXiv:hep-ph/0205023.

15. W. Bernreuther, A. Brandenburg, Z. G. Si and P. Uwer, Phys. Rev. Lett. 87 (2001) 242002 arXiv:hep-ph/0107086.

16. T. Affolder et al. [CDF Collaboration], Phys. Rev. Lett. 84 (2000) 216 arXiv:hep-ex/9909042.

17. S. D. Ellis, Z. Kunszt and D. E. Soper, Phys. Rev. Lett. 64 (1990) 2121.

18. W.T. Giele, E.W.N. Glover and D.A. Kosower, Phys. Rev. Lett. 73 (1994) 2019 arXiv:hep-ph/9403347.

19. Z. Nagy, Phys. Rev. Lett. 88 (2002) 122003 arXiv:hep-ph/0110315.

20. W.T. Giele, E.W.N. Glover and D.A. Kosower, Nucl. Phys. B 403 (1993) 633 arXiv:hep-ph/9302225.

21. J. Campbell and R.K. Ellis, Phys. Rev. D 65 (2002) 113007 arXiv:hep$\mathrm{ph} / 0202176$.

22. B. Abbott et al. [D0 Collaboration], Phys. Rev. Lett. 82 (1999) 2451 arXiv:hep-ex/9807018.

23. S.D. Ellis and D.E. Soper, Phys. Rev. D 48 (1993) 3160 arXiv:hepph/9305266 ; S. Catani, Y. L. Dokshitzer, M. H. Seymour and B. R. Webber, Nucl. Phys. B 406 (1993) 187.

24. G.C. Blazey et al., "Run II jet physics,", Proceedings of the Workshop "QCD and Weak Boson Physics in Run II", Fermilab, 1999, arXiv:hep-ex/0005012.

25. T. Affolder et al. [CDF Collaboration], Phys. Rev. Lett. 88 (2002) 042001 arXiv:hep-ex/0108034.

26. K. Long, plenary talk at ICHEP02, Amsterdam, 2002.

27. E.W.N. Glover, these proceedings.

28. W.T. Giele and E.W.N. Glover, Phys. Rev. D 46 (1992) 1980; S. Catani and M. H. Seymour, Nucl. Phys. B 485 (1997) 291 [Erratum-ibid. B 510 (1997) 503] arXiv:hep-ph/9605323.

29. Z. Bern, L.J. Dixon, D.C. Dunbar and D.A. Kosower, Nucl. Phys. B 425 (1994) 217 arXiv:hep-ph/9403226; D.A. Kosower, Nucl. Phys. B 552 (1999) 319 arXiv:hep-ph/9901201|; D.A. Kosower and P. Uwer, Nucl. Phys. B 563 (1999) 477 [arXiv:hep-ph/9903515]; Z. Bern, V. Del Duca and C.R. Schmidt, Phys. Lett. B 445 (1998) 168 arXiv:hep-ph/9810409; Z. Bern, V. Del Duca, 
W.B. Kilgore and C.R. Schmidt, Phys. Rev. D 60 (1999) 116001 arXiv:hepph/9903516; S. Catani and M. Grazzini, Nucl. Phys. B 591 (2000) 435 arXiv:hep-ph/0007142.

30. J.M. Campbell and E.W.N. Glover, Nucl. Phys. B527 (1998) 264 arXiv:hepph/9710255; S. Catani and M. Grazzini, Phys. Lett. B446 (1999) 143 arXiv:hep-ph/9810389; Nucl. Phys. B570 (2000) 287 arXiv:hepph/9908523; F.A. Berends and W.T. Giele, Nucl. Phys. B313 (1989) 595.

31. A. Gehrmann-De Ridder, T. Gehrmann and E.W.N. Glover, Phys. Lett. B 414 (1997) 354 arXiv:hep-ph/9705305; A. Gehrmann-De Ridder and E.W.N. Glover, Nucl. Phys. B 517 (1998) 269 arXiv:hep-ph/9707224].

32. S. Laporta, Int. J. Mod. Phys. A 15 (2000) 5087 arXiv:hep-ph/0102033.

33. F.V. Tkachov, Phys. Lett. 100B (1981) 65; K.G. Chetyrkin and F.V. Tkachov, Nucl. Phys. B 192 (1981) 159.

34. T. Gehrmann and E. Remiddi, Nucl. Phys. B 580 (2000) 485 arXiv:hep$\mathrm{ph} / 9912329$.

35. V.A. Smirnov, Phys. Lett. B 460 (1999) 397 arXiv:hep-ph/9905323; V.A. Smirnov and O.L. Veretin, Nucl. Phys. B 566 (2000) 469 arXiv:hepph/9907385; J.B. Tausk, Phys. Lett. B 469 (1999) 225 arXiv:hepph/9909506; C. Anastasiou, T. Gehrmann, C. Oleari, E. Remiddi and J.B. Tausk, Nucl. Phys. B 580 (2000) 577 arXiv:hep-ph/0003261; T. Gehrmann and E. Remiddi, Nucl. Phys. B (Proc. Suppl.) 89 (2000) 251 arXiv:hepph/0005232]; C. Anastasiou, J.B. Tausk and M.E. Tejeda-Yeomans, Nucl. Phys. B (Proc. Suppl.) 89 (2000) 262 arXiv:hep-ph/0005328.

36. T. Gehrmann and E. Remiddi, Nucl. Phys. B 601 (2001) 248 arXiv:hepph/0008287 and 601 (2001) 287 arXiv:hep-ph/0101124.

37. T. Gehrmann, arXiv:hep-ph/0210157.

38. Z. Bern, L. Dixon and A. Ghinculov, Phys. Rev. D 63 (2001) 053007 arXiv:hep-ph/0010075.

39. C. Anastasiou, E.W.N. Glover, C. Oleari and M.E. Tejeda-Yeomans, Nucl. Phys. B 601 (2001) 318 arXiv:hep-ph/0010212; 601 (2001) 347 arXiv:hepph/0011094; 605 (2001) 486 arXiv:hep-ph/0101304; E.W.N. Glover, C. Oleari and M.E. Tejeda-Yeomans, Nucl. Phys. 605 (2001) 467 arXiv:hepph/0102201: Z. Bern, A. De Freitas and L. Dixon, JHEP 0203 (2002) 018 arXiv:hep-ph/0201161.

40. Z. Bern, A. De Freitas and L. J. Dixon, JHEP 0109 (2001) 037 arXiv:hepph/0109078]; C. Anastasiou, E.W.N. Glover and M.E. Tejeda-Yeomans, Nucl. Phys. B 629 (2002) 255 arXiv:hep-ph/0201274.

41. Z. Bern, A. De Freitas, L.J. Dixon, A. Ghinculov and H.L. Wong, JHEP 0111 (2001) 031 arXiv:hep-ph/0109079; T. Binoth, E.W.N. Glover, P. Marquard and J.J. van der Bij, JHEP 0205 (2002) 060 arXiv:hep-ph/0202266.

42. L.W. Garland, T. Gehrmann, E.W.N. Glover, A. Koukoutsakis and E. Remiddi, Nucl. Phys. B 627 (2002) 107 arXiv:hep-ph/0112081 and 642 (2002) 227 arXiv:hep-ph/0206067.

43. S. Moch, P. Uwer and S. Weinzierl, arXiv:hep-ph/0207043.

44. S. Moch, P. Uwer and S. Weinzierl, J. Math. Phys. 43 (2002) 3363 arXiv:hep-ph/0110083; S. Weinzierl, Comput. Phys. Commun. 145 (2002) 357 arXiv:math-ph/0201011.

45. T. Gehrmann and E. Remiddi, Nucl. Phys. B 640 (2002) 379 arXiv:hep$\mathrm{ph} / 0207020$. 
46. S. Catani, Phys. Lett. B 427 (1998) 161 arXiv:hep-ph/9802439; G. Sterman and M.E. Tejeda-Yeomans, arXiv:hep-ph/0210130.

47. F. Caravaglios and M. Moretti, Phys. Lett. B 358 (1995) 332 arXiv:hep$\mathrm{ph} / 9507237$.

48. F. Caravaglios, M. L. Mangano, M. Moretti and R. Pittau, Nucl. Phys. B 539 (1999) 215 arXiv:hep-ph/9807570.

49. M. L. Mangano, M. Moretti, F. Piccinini, R. Pittau and A. D. Polosa, arXiv:hep-ph/0206293.

50. S. Catani, F. Krauss, R. Kuhn and B. R. Webber, JHEP 0111 (2001) 063 arXiv:hep-ph/0109231.

51. F. Krauss, JHEP 0208 (2002) 015 arXiv:hep-ph/0205283

52. B. Pötter, Phys. Rev. D 63 (2001) 114017 arXiv:hep-ph/0007172;;

M. Dobbs, Phys. Rev. D 65 (2002) 094011 [arXiv:hep-ph/0111234];

S. Frixione and B. R. Webber, JHEP 0206 (2002) 029 arXiv:hep-ph/0204244.

53. B. Pötter and T. Schorner, Phys. Lett. B 517 (2001) 86 arXiv:hep$\mathrm{ph} / 0104261$;

S. Frixione and B. R. Webber, arXiv:hep-ph/0207182.

54. E.W.N. Glover and A.G. Morgan, Z. Phys. C 62 (1994) 311.

55. S. Catani, M. Fontannaz, J. P. Guillet and E. Pilon, JHEP 0205 (2002) 028 arXiv:hep-ph/0204023.

56. D. Buskulic et al. [ALEPH Collaboration], Z. Phys. C 69 (1996) 365.

57. K. Ackerstaff et al. [OPAL Collaboration], Eur. Phys. J. C 2 (1998) 39 arXiv:hep-ex/9708020.

58. A. Gehrmann-De Ridder and E.W.N. Glover, Eur. Phys. J. C 7 (1999) 29 arXiv:hep-ph/9806316.

59. A. Gehrmann-De Ridder, G. Kramer and H. Spiesberger, Eur. Phys. J. C 11 (1999) 137 arXiv:hep-ph/9907511.

60. S. Lee, these proceedings.

61. R. Harlander, these proceedings.

62. T. Binoth, J.P. Guillet, E. Pilon and M. Werlen, Eur. Phys. J. C 16 (2000) 311 arXiv:hep-ph/9911340.

63. Z. Bern, L. Dixon and C. Schmidt, Phys. Rev. D 66 (2002) 074018 arXiv:hep$\mathrm{ph} / 0206194$.

64. T. Binoth, J. P. Guillet, E. Pilon and M. Werlen, Phys. Rev. D 63 (2001) 114016 arXiv:hep-ph/0012191.

65. T. Binoth, J. P. Guillet, E. Pilon and M. Werlen, Eur. Phys. J. C 24 (2002) 245 arXiv:hep-ph/0111043.

66. R. Hamberg, W. L. van Neerven and T. Matsuura, Nucl. Phys. B 359 (1991) 343 [Erratum-ibid. B 644 (2002) 403].

67. R.V. Harlander and W.B. Kilgore, Phys. Rev. Lett. 88 (2002) 201801 arXiv:hep-ph/0201206.

68. A.D. Martin, R.G. Roberts, W.J. Stirling and R.S. Thorne, Eur. Phys. J. C 14 (2000) 133 arXiv:hep-ph/9907231, and R. Thorne, these proceedings.

69. S. Moch, J. A. Vermaseren and A. Vogt, Nucl. Phys. B 646 (2002) 181 arXiv:hep-ph/0209100.

70. M. Dittmar, these proceedings.

71. G. Altarelli, R.K. Ellis and G. Martinelli, Nucl. Phys. B 157 (1979) 461.

72. R.V. Harlander, Phys. Lett. B 492 (2000) 74 arXiv:hep-ph/0007289; S. Catani, D. de Florian and M. Grazzini, JHEP 0105 (2001) 025 arXiv:hepph/0102227]; R.V. Harlander and W.B. Kilgore, Phys. Rev. D 64 (2001) 013015 arXiv:hep-ph/0102241. 
73. C. Anastasiou and K. Melnikov, Nucl. Phys. B 646 (2002) 220 arXiv:hep$\mathrm{ph} / 0207004$.

74. R.V. Harlander and W.B. Kilgore, JHEP 0210 (2002) 017 arXiv:hepph/0208096; C. Anastasiou and K. Melnikov, arXiv:hep-ph/0208115.

75. R.K. Ellis, G. Martinelli and R. Petronzio, Nucl. Phys. B 211 (1983) 106; D. de Florian, M. Grazzini and Z. Kunszt, Phys. Rev. Lett. 82 (1999) 5209 arXiv:hep-ph/9902483; V. Ravindran, J. Smith and W.L. Van Neerven, Nucl. Phys. B 634 (2002) 247 arXiv:hep-ph/0201114.

76. J. C. Collins, D. E. Soper and G. Sterman, Nucl. Phys. B 250 (1985) 199.

77. J. Kodaira and L. Trentadue, Phys. Lett. B 112 (1982) 66; S. Catani, E. D'Emilio and L. Trentadue, Phys. Lett. B 211 (1988) 335.

78. C. T. Davies and W. J. Stirling, Nucl. Phys. B 244 (1984) 337.

79. D. de Florian and M. Grazzini, Phys. Rev. Lett. 85 (2000) 4678 arXiv:hep$\mathrm{ph} / 0008152$

80. W. Giele et al., "The QCD/SM working group: Summary report,", Proceedings of Les Houches Workshop on "Physics at TeV Colliders", 2001, arXiv:hep$\mathrm{ph} / 0204316$.

81. C. F. Berger, arXiv:hep-ph/0209107.

82. S. Catani, D. de Florian and M. Grazzini, Nucl. Phys. B 596 (2001) 299 arXiv:hep-ph/0008184.

83. D. de Florian and M. Grazzini, Nucl. Phys. B 616 (2001) 247 arXiv:hep$\mathrm{ph} / 0108273$.

84. E. Laenen, G. Sterman and W. Vogelsang, Phys. Rev. D 63 (2001) 114018 arXiv:hep-ph/0010080.

85. E. Laenen, G. Sterman and W. Vogelsang, Phys. Rev. Lett. 84 (2000) 4296 arXiv:hep-ph/0002078; A. Kulesza, G. Sterman and W. Vogelsang, Phys. Rev. D 66 (2002) 014011 arXiv:hep-ph/0202251. 